\title{
Diseño, desarrollo y pruebas de un electrocardiógrafo virtual utilizando LabVIEW
}

\author{
Guadalupe Monrroy Andrade, Dayra Iovana Zepeda González, \\ Saida Anai Ricario Vazquez, Roberto Alejandro Ledesma Camacho, \\ María Cosio León \\ Universidad Autónoma de Baja California, Facultad de Ingeniería, Arquitectura y Diseño, \\ México \\ \{guadalupe.monroy, dayra.zepeda, saida.ricario, roberto.ledesma\}@uabc.edu.mx
}

\begin{abstract}
Resumen. En la actualidad los padecimientos cardiovasculares se encuentran entre las principales enfermedades, que afectan a millones de personas en el mundo; por ello la importancia de desarrollar herramientas que permitan a especialistas su detección temprana. El presente trabajo tiene como objetivo el diseño, desarrollo y prueba de un electrocardiograma (ECG) virtual, que permita la adquisición, procesamiento, despliegue y almacenamiento de la actividad cardíaca. Para su desarrollo se hace uso de diferentes herramientas de software y hardware provistas por la compañía de National Instruments: LabVIEW (Laboratory Virtual Instrument Engineering Workbench), NI myDAQ, NI ELVIS II+ software-hardware y NI LabVIEW Advanced Signal Processing Toolkit. Como resultado se logró la implementación completa del instrumento virtual y con ello, la posibilidad de trabajar con datos reales o datos simulados.
\end{abstract}

Palabras clave: Electrocardiograma, LabVIEW, NI LabVIEW advanced signal processing toolkit.

\section{Introducción}

Según el Instituto Nacional de Estadística y Geografía (2010), los problemas cardiovasculares se encuentran en la primera posición dentro de los padecimientos con mayor tasa de mortalidad en el país, debido a la falta de diagnóstico temprano y a que sólo una baja proporción de los individuos con hipertensión acude a los servicios de salud en busca de atención; por otro lado, a los que son beneficiados con servicios de salud, su tratamiento médico es de baja efectividad, como se explica en [1]. La Fig.1, muestra las epidemiologías con mayor índice de defunciones, encontradas durante el censo del INEGI 2010.

Uno de los métodos para la detección temprana de dichos padecimientos es el electrocardiograma (ECG), el cual consiste en el registro gráfico, en función del tiempo de las variaciones de potencial eléctrico, generadas por el conjunto de células cardiacas; este registro es obtenido en la superficie corporal por medio de electrodos.

El ECG es la técnica más utilizada para el estudio electrofisiológico del corazón, así como para la determinación de anomalías y padecimientos crónicos en el 
funcionamiento cardiaco. Desde sus inicios el ECG ha sido interpretado a partir de la morfología de las ondas y complejos que componen el ciclo cardíaco y de las mediciones de intervalos de tiempo entre las diferentes ondas, complejos y segmentos (ver Fig. 3) [2].

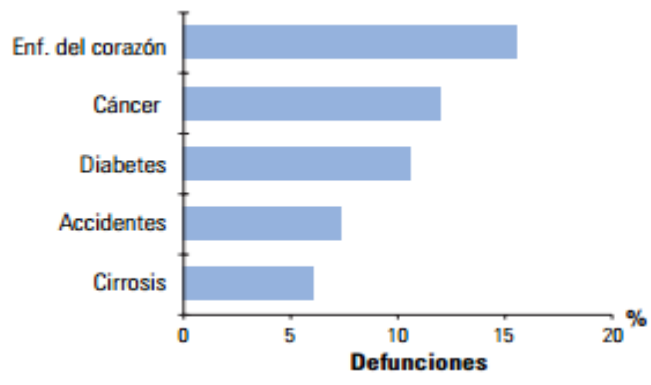

Fig. 1. Principales epidemiologias causantes de mortalidad en México [1].

Por lo anterior, es necesario el diseño de dispositivos de monitorización de señales fisiológicas que provean soluciones que permitan al personal médico realizar diagnósticos actualizados del paciente. En este trabajo se propone un instrumento virtual que incluye etapas de adquisición - no se incluye el detalle de componentes electrónicos-, tratamiento, presentación y almacenamiento de la señal del ECG.

Todos los elementos de software aquí descritos pueden utilizarse en tarjetas de desarrollo como la NI myRIO, hardware propuesto por la compañía NI LabVIEW (Laboratory Virtual Instrument Engineering Workbench), plataforma con herramientas que permiten el procesamiento del ECG, el electroencefalograma (EEG), el electromiógrafo (EMG), y otras señales fisiológicas [3]. NI LabVIEW se auxilia de otros elementos de hardware como el NI myDAQ, el cual es un dispositivo que brinda la habilidad para adquirir señales digitales, analógicas y de sonido; y el NI ELVIS II+, hardware de soporte que aporta instrumentos virtuales de laboratorio básicos como: osciloscopio, multímetro, fuentes de poder, entre otros.

\subsection{Actividad cardíaca}

\section{Propiedades Eléctricas del Corazón}

Las células del corazón ubicadas en el miocardio son llamadas miocardiocitos, éstas no requieren de estímulos externos para generar una respuesta de contracción, capacidad de contraerse y despolarizarse rítmicamente sin inervación del miocardio; esto se denomina como ritmo cardiaco.

En el tejido del corazón se distinguen dos tipos de fibras para su funcionamiento:

- Fibras automáticas, capaces de generar y conducir el potencial de acción, se caracterizan por establecer una respuesta lenta al impulso bioeléctrico.

- Fibras de trabajo o auriculares, requieren un estímulo para su excitación. Sin embargo, en algunas ocasiones pueden trabajar de manera automática, pero a un ritmo más lento que las primeras. 
La diferencia de ritmo que posee cada tipo de fibra es lo que determina que a las fibras auriculares (nodo Sinusal, ver Fig. 2) se les denomine células marcapaso [4].

\section{Sistema Eléctrico del Corazón}

La propagación del potencial de acción cardíaco se difunde a través de un sistema de excitación y conducción. Como se observa en la Fig. 2, el sistema se compone del Haz de His, fibras de Purkinje, Nódulo sinusal y Nódulo auriculoventricular. El ciclo inicia con la despolarización en el Nódulo sinusal, que se expande por todas las fibras auriculares, conduciendo hasta el Nódulo auriculoventricular, donde la conducción del pulso es lento. Dada la geometría de las células que forman al Nódulo, después de este lapso, el pulso se propaga rápidamente hacia el resto del músculo cardiaco [5].

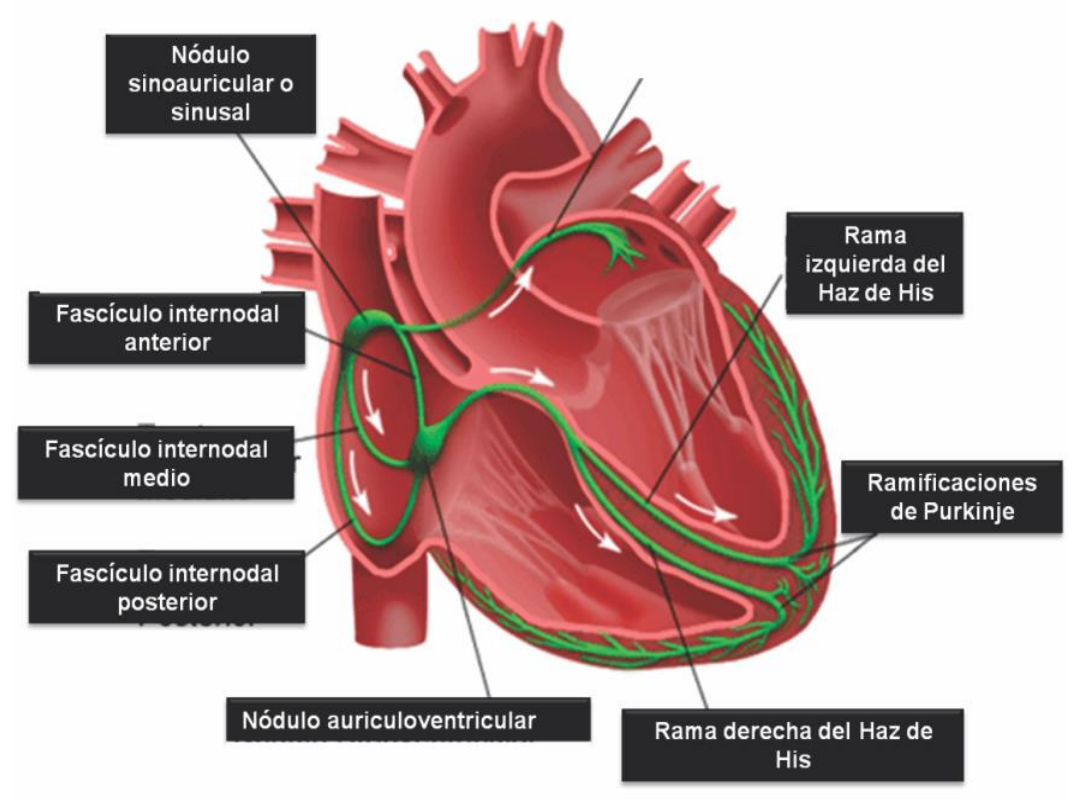

Fig. 2. Nodo Sinusal.

\section{Potencial de Acción Cardíaco}

La contracción del músculo cardiaco, es generada a partir de una respuesta eléctrica en la membrana denominada potencial de acción cardíaca -el potencial eléctrico en las fibras cardíacas oscila entre 150 y $300 \mathrm{~ms}^{-}$. Este intervalo tiene importantes consecuencias funcionales, se encarga de garantizar que el músculo no pueda volver a excitarse antes de terminar la contracción cardiaca [6].

Bajo condiciones de reposo una célula cardiaca posee carga negativa en su interior y positiva en su exterior, el equilibrio es mantenido por la permeabilidad selectiva de la membrana a cationes, y posteriormente a la actividad de la bomba Potasio y Sodio (Na-K), la diferencia de cargas a ambos lados de la membrana se denomina potencial de reposo. 
Las células del Nódulo sinusal y Nódulo auriculoventricular presentan cambios de potencial rítmicos en la membrana del tejido cardiaco, las fases en las que se desarrolla la actuación son [7]:

- Fase de reposo inestable. El potencial de membrana se despolariza hasta generar un potencial de acción, lo que permite el paso de cargas positivas a través de ella. Este proceso de despolarización se conoce como potencial marcapasos o despolarización diastólica.

- Fase de despolarización. Debido a la entrada de cationes provenientes del exterior de la membrana.

- Fase de repolarización. El potencial de acción se conduce por las fibras auriculares hasta llegar al Haz de His.

Ondas, Intervalos y Segmentos del Electrocardiograma

El electrocardiograma habitual consta de 5 ondas: Q, P, R, S y T; además de segmentos que expresan momentos de polarización y despolarización auricular y ventricular.

Como se muestra en la Fig. 3, la onda $\mathrm{P}$ es el resultado de la despolarización auricular, la cual tiene una amplitud de $3 \mathrm{mV}$ y duración máxima de 0.10 segundos. Inmediatamente después, aparecen $\mathrm{Q}, \mathrm{R}$ y $\mathrm{S}$, integrando el complejo ventricular, por la propagación de la onda de excitación a la musculatura de ambos ventrículos con longitud máxima de 0.08 segundos en niños y 0.10 segundos en adultos [4]. El segmento S-T, es el intervalo de repolarización ventricular con longitud máxima de 0.9 segundos en niños y 0.12 segundos en adultos; por último aparece la onda T, para la repolarización ventricular con una duración de 0.1 segundos en niños y 0.3 segundos en adultos [8].

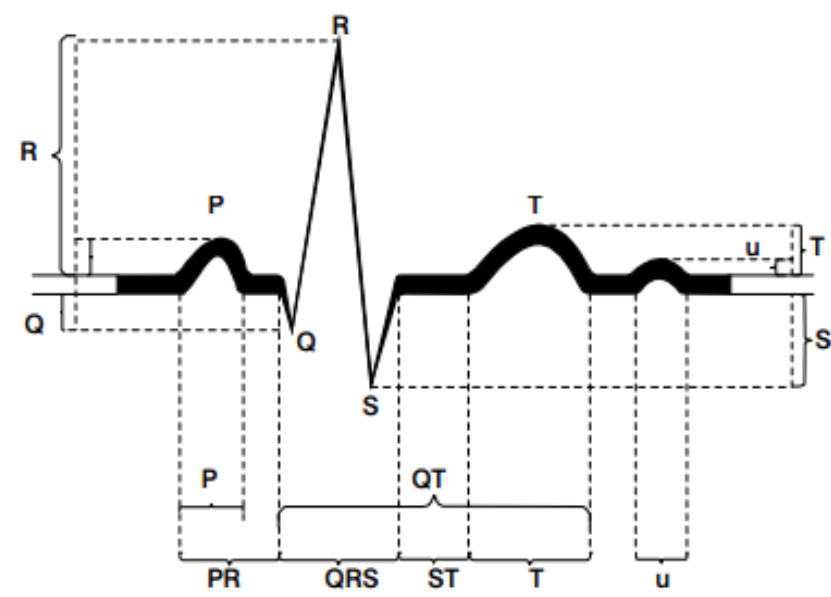

Fig. 3. Se muestra el compartimento normal de los distintos elementos de ECG [4].

En la Tabla 1, se observa que la frecuencia cardiaca disminuye en el individuo con relación al aumento de su edad hasta llegar a la edad de 10 años, donde en individuos sanos es 60-100 latidos por minuto (lat/min.) [9]. 
Tabla 1. Estadística de frecuencia cardiaca normal en niños y adultos [8].

\begin{tabular}{cc}
\hline PACIENTE & FRECUENCIAS \\
\hline Bebés (1-11 meses) & $80-160 \mathrm{lat} / \mathrm{min}$ \\
Niños (1-2 años) & $80-130 \mathrm{lat} / \mathrm{min}$ \\
Niños (3-4 años) & $80-120 \mathrm{lat} / \mathrm{min}$ \\
Niños (5-6 años) & $75-115 \mathrm{lat} / \mathrm{min}$ \\
Niños (7-9 años) & $70-110 \mathrm{lat} / \mathrm{min}$ \\
Niños de 10 años y adultos & $60-100 \mathrm{lat} / \mathrm{min}$ \\
\hline
\end{tabular}

La duración del segmento Q-T, es analizada con respecto a la frecuencia cardiaca para la determinación de padecimientos congénitos y anomalías crónicas. En la Tabla 2 , se observa que la duración del segmento Q-T aumenta con respecto a la edad del paciente y la frecuencia cardíaca.

Tabla 2. Duración del espacio Q-T en relación con la frecuencia cardiaca [4].

\begin{tabular}{|c|c|c|c|c|c|c|}
\hline FRECUENCIA & $\begin{array}{c}0-1 \\
\text { MES }\end{array}$ & $\begin{array}{c}1-6 \\
\text { MESES }\end{array}$ & $\begin{array}{c}6 \mathrm{M} \\
1 \mathrm{AN \tilde {N }}\end{array}$ & $\begin{array}{c}\mathbf{1 - 3} \\
\text { AÑNOS }\end{array}$ & $\begin{array}{c}\mathbf{3 - 8} \\
\text { Ã̃NOS }\end{array}$ & ADULTO \\
\hline$<60$ & & & & & $0.16-0.18$ & $0.17-0.21$ \\
\hline $60-80$ & & & & $0.14-0.16$ & $0.15-0.17$ & $0.16-0.21$ \\
\hline $80-100$ & & & & $0.13-0.16$ & $0.15-0.16$ & $0.15-0.20$ \\
\hline $100-120$ & $0.10-0.12$ & $0.10-0.14$ & $0.11-0.14$ & $0.13-0.15$ & $0.14-0.15$ & $0.15-0.19$ \\
\hline $120-140$ & $0.10-0.11$ & $0.10-0.13$ & $0.11-0.13$ & $0.12-0.14$ & & $0.15-0.18$ \\
\hline $140-160$ & $0.09-0.11$ & $0.10-0.12$ & $0.10-0.12$ & & & 0.17 \\
\hline$>180$ & 0.09 & $0.09-0.11$ & & & & \\
\hline
\end{tabular}

El ECG genera un registro gráfico, que muestra el comportamiento de la actividad eléctrica del músculo cardiaco; la velocidad de registro es de $25 \mathrm{~mm}$ por segundo, por lo que los resultados son: a) cada cuadrícula pequeña $=1 \mathrm{~mm}$; b) cada cuadro mayor $=$ $5 \mathrm{~mm}$; c) 5 cuadros mayores $=25 \mathrm{~mm}$. Si 300 cuadros grandes son un minuto hay que dividir 300 entre el número de cuadros que existen entre dos latidos, sabiendo esto, se pueden calcular la frecuencia cardiaca midiendo el intervalo RR [2].

\subsection{La transformada de Wavelet para el análisis del ECG}

La transformada de Wavelet, vista como un proceso matemático, es una operación lineal que descompone una señal compleja en bloques elementales que aparecen en diferentes escalas o resoluciones [10].

Existen importantes diferencias entre el análisis de Fourier y el de Wavelet. La primera diferencia consiste en que la transformada de Fourier produce cambios en todo el dominio del tiempo; por otro lado, las Waveletes son locales en dos dimensiones: Frecuencialescala (vía dilataciones) y en el Tiempo (vía translaciones); esta forma de operar es una ventaja para algunos problemas, como en el análisis de señales fisiológicas cuyo comportamiento es pseudo periódico. 
La segunda diferencia, es que muchas clases de funciones pueden ser representadas por Waveletes en una forma más compacta; por ejemplo, funciones con discontinuidades y funciones con picos muy pronunciados, comportamiento que sigue la señal del ECG en la Fig. 4.

La selección de la Wavelet está fuertemente relacionada con las características de la señal de estudio; por lo tanto, el punto clave es encontrar la función base que mejor se adecue a la señal y que se identificará como Wavelet madre; es importante mencionar que sólo aquellas funciones que satisfagan las dos condiciones siguientes pueden ser consideradas como tal [11]: 1. Ser oscilatorio (área total igual a cero); 2. Decaer a cero rápidamente.

Para este trabajo se eligió la familia de Waveletes de Daubechies, en específico la número 6 (db6), la cual se ajusta de mejor forma a la señal generada por el ECG como se observa en las Fig. 4a, 4b, 4c, 4d.

a) Función de escalado
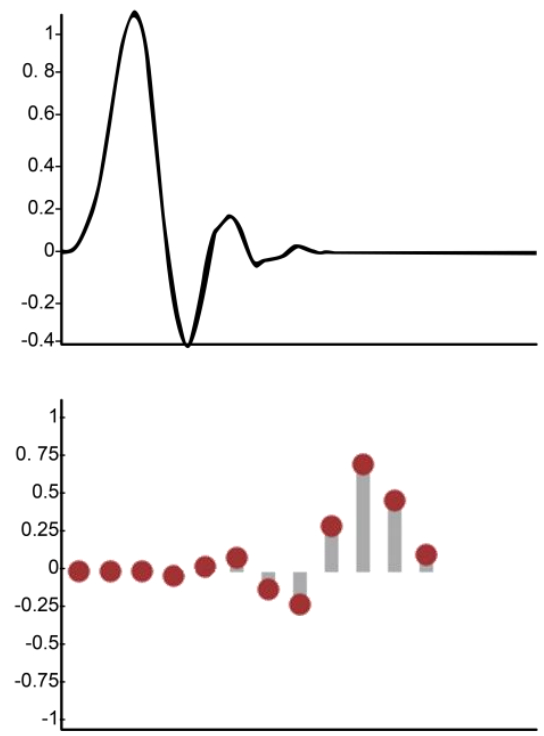

c) Descomposición del filtro pasa bajas b) Función Wavelet
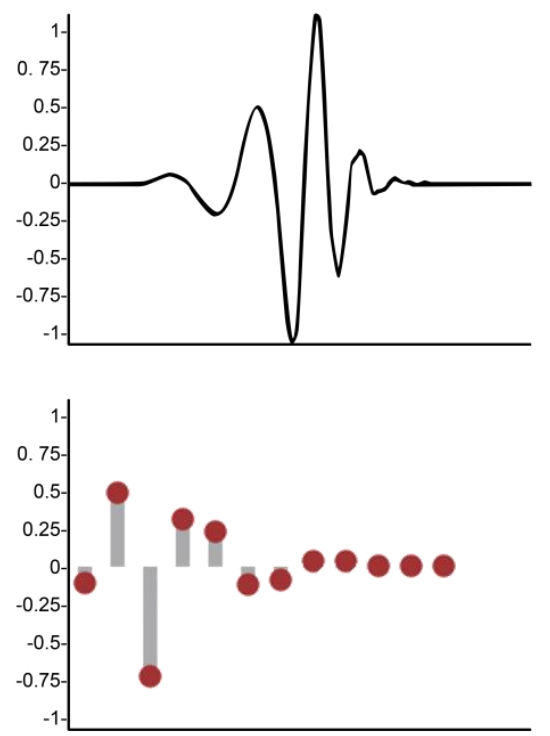

d) Descomposición del filtro pasa altas

Fig. 4. Familia db6 de Waveletes Daubechies [11].

\section{Diseño y desarrollo del ECG en LabVIEW}

Una primera aproximación al diseño del instrumento virtual es el que se muestra en la Fig 5, en el cual se generaron los primeros resultados. Este diagrama muestra dos componentes Express (Simulate ECG y Filter), además de dos pantallas de despliegue de la señal ECG, una sin tratar, y la segunda ya tratada; todos ellos dentro de un Ciclo While. El programa antes descrito permite limpiar una señal generada mediante el $N I$ 
Biomedical Toolkit, la cual tiene ruido blanco y ruido de $60 \mathrm{~Hz}$. Se propone el uso de un filtro pasa banda de tipología elíptica de 4to. orden. Este filtro tiene una frecuencia inferior de $0.01 \mathrm{~Hz}$ y frecuencia de corte superior de $50 \mathrm{~Hz}$, la selección de estos parámetros se hace considerando las características de la señal ECG [12].

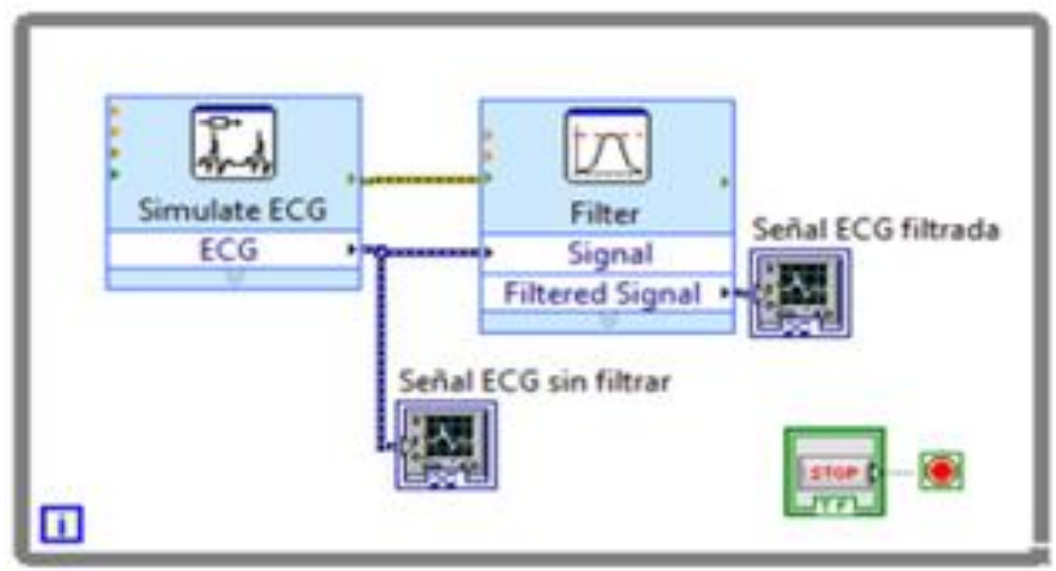

Fig. 5. Diseño de filtros. Filtro pasa banda de tipología elíptica de 4to orden.

Una vez que la señal es manipulada por el filtro pasa banda, como se observa en la Fig. 6, el ruido es eliminado y se pueden distinguir con mayor precisión los componentes básicos de una señal ECG típica. Para obtener una buena señal de ECG sin ruido y poder analizarla para su posterior diagnóstico, se implementaron componentes de LabVIEW como WA Detrend, el cual elimina la tendencia a partir de señales $1 \mathrm{D}$, para ello se ajustan los coeficientes de aproximación a ceros.

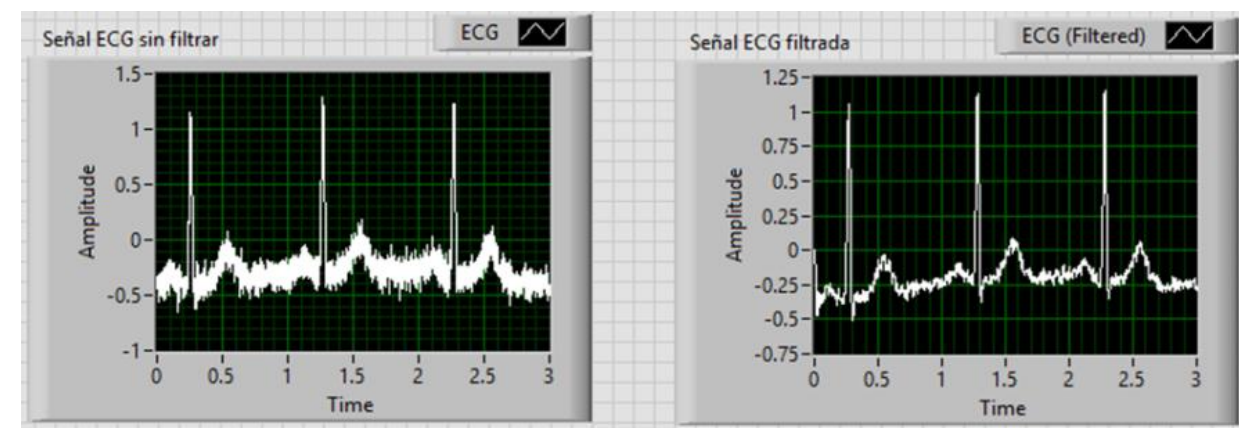

Fig. 6. Señal antes y después del filtrado.

$$
\text { Trend level }=\frac{\log _{2} 2 T}{\log _{2} N}
$$

El módulo WA Detrend tiene la opción para seleccionar el tipo de Wavelet utilizado para el análisis (ver Fig.7); cuyos datos tienen una duración de 10 segundos y 5000 puntos en el muestreo total; por lo que el nivel de tendencia es 0.35 , de acuerdo con la 
Ecuación 1, descrita y justificada en [13]. Donde T, es la duración del muestreo y N, es el número de puntos de muestreo.
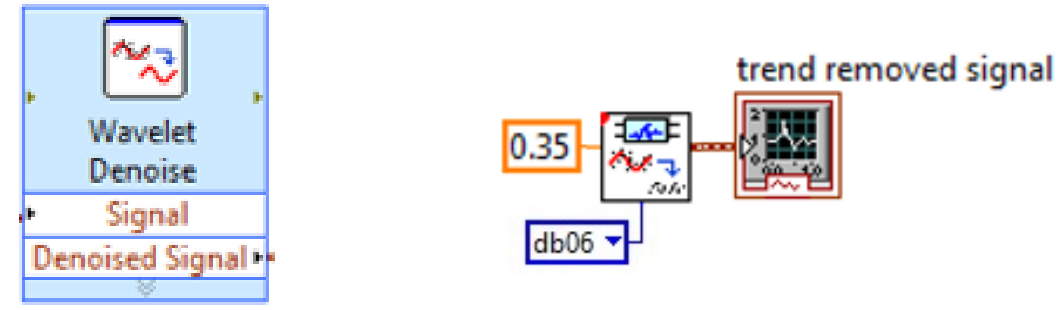

Fig. 7. Express Wavelet Detrend y su configuración.

Se toma el mismo dato de tendencia en este desarrollo, ya que se trata de una señal de ECG Wavelet discreta. También se implementa el módulo Waveform Peak Detection (ver Fig. 8), el cual encuentra los picos y valles de la señal. A éste se le agrega un Threshold para filtrar los picos que se desean observar.

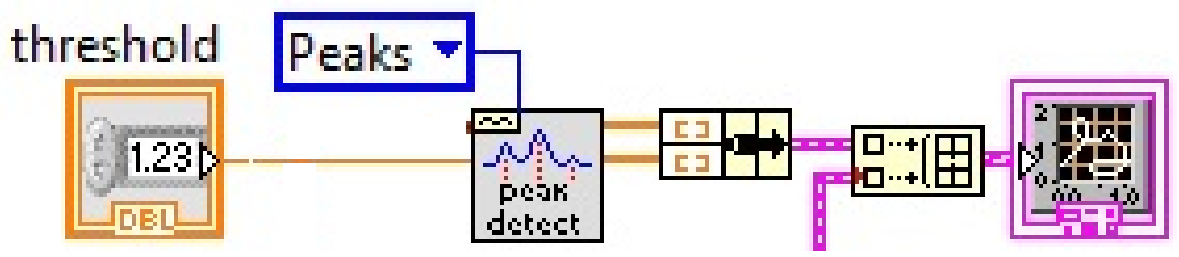

Fig. 8. Esquema de Waverform Peak Detecion.

El módulo Express Waveform Peak Detection se conecta a un Bundle (estructura que une dos o más lecturas en un solo paquete), seguido de ello a Buid Array (construye un arreglo con las lecturas procesadas) hasta el despliegue de la información en un Waveform Graph, el cual nos mostrará gráficamente los picos de la señal. La ¡Error! No se encuentra el origen de la referencia. 9 muestra el diagrama de bloques para el análisis de la señal de ECG incluyendo Express Waveform Peak Detection.

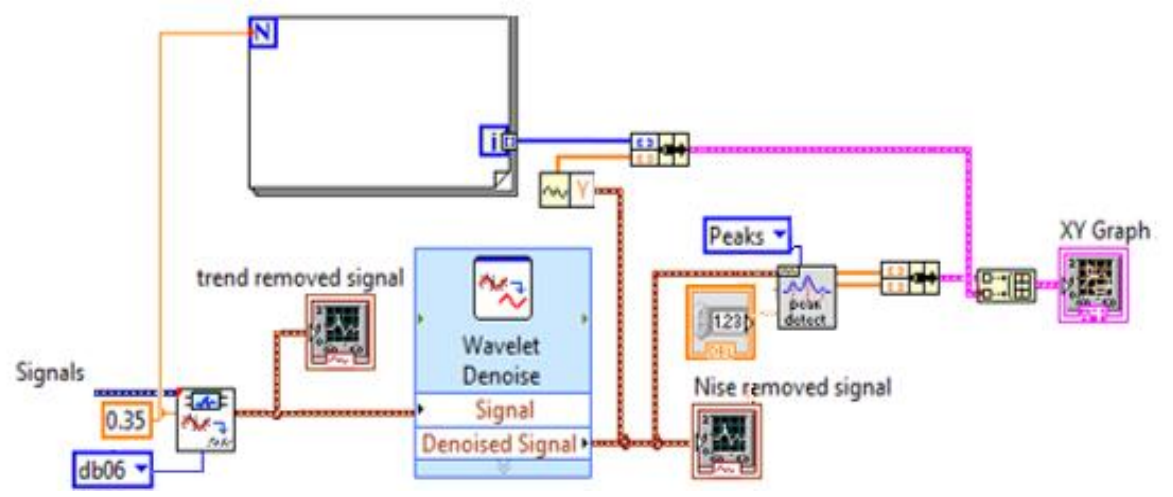

Fig. 9. Integración de Express Waveform Peak Detection 
También se implementó el módulo Express Amplitude and Level Measurements (ver Fig. 10), el cual realiza mediciones de tensiones de una señal y contiene las opciones mostradas en la Tabla 3.

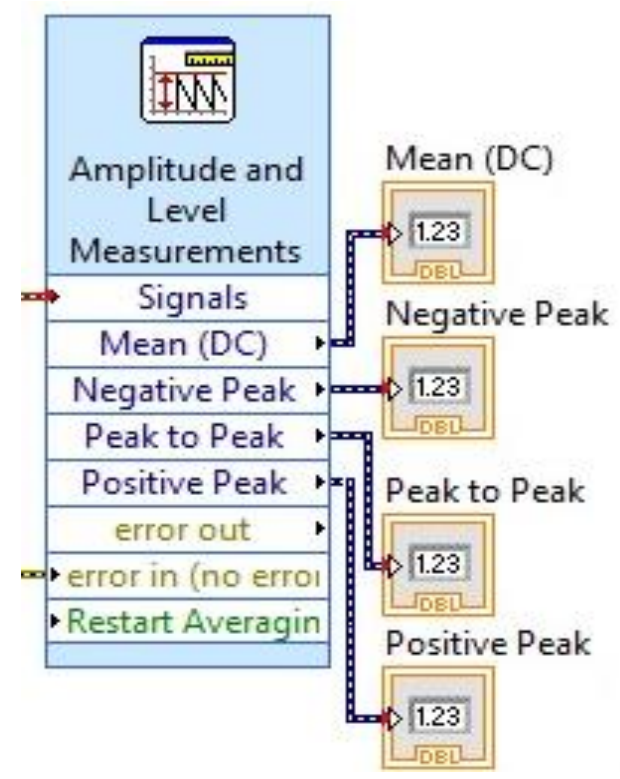

Fig. 10. Express Amplitude and level measurements.

Se integraron los VI generados durante el desarrollo del trabajo, obteniendo el siguiente VI final (ver Fig. 11).

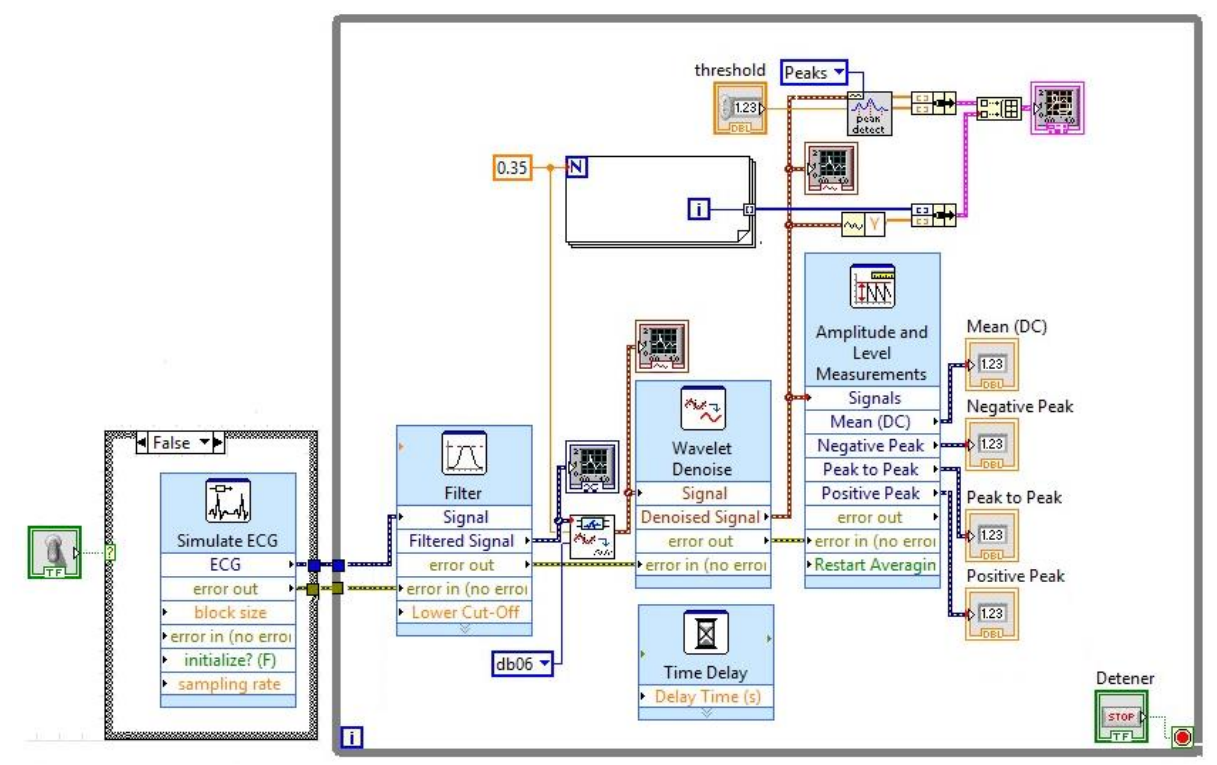

Fig. 11. Implementación final de ECG virtual. 
Guadalupe Monrroy, Dayra Zepeda, Saida Ricario, Roberto Ledesma y María Cosio

Tabla 3. Express Amplitude and Level measurements.

\begin{tabular}{ll}
\hline Característica & Descripción \\
\hline La media (DC) & Adquiere una medición continua de señales. \\
$R M S$ & Calcula el valor cuadrático medio de señales. \\
Apply window & Aplica una ventana baja de los lóbulos laterales de señales. \\
Positive peak & Mide el pico más positivo de las señales. \\
Negative peak & Mide el pico más negativo de las señales. \\
Peak to peak & Mide distancia entre pico y pico. \\
\hline
\end{tabular}

En este diseño se observa la posibilidad de adquirir la señal a procesar de dos fuentes, para ello se implementa una estructura tipo Case, con las siguientes opciones: False, usando el módulo NI Biomedical Toolkit; en el cual se generó una señal de ECG con ruido blanco y ruido de $60 \mathrm{~Hz}$, para simular las condiciones reales de adquisición de señales. La opción True, a través del módulo DAQ Assistant, para el sensado de la señal en tiempo real. Las etapas posteriores del sistema, consisten en el tratamiento de la señal mediante filtros, procesamiento de la señal y obtención de valores estadísticos.

\subsection{Resultados}

En la Fig. 12, se muestra el procesamiento de una señal de ECG con la transformada de Wavelet db06; los datos fueron obtenidos de bases de datos clínicos de la página www.physionet.org.
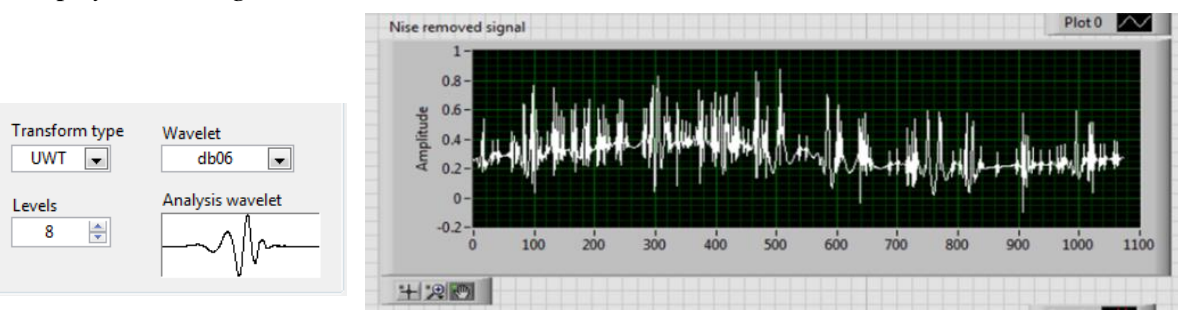

Fig. 12. Señal con el db06.

Se realizaron pruebas considerando dos configuraciones para las Wavelet madre, db12 en Fig. 13; db2 en Fig. 14. Las cuales no se ajustaban a la señal original.
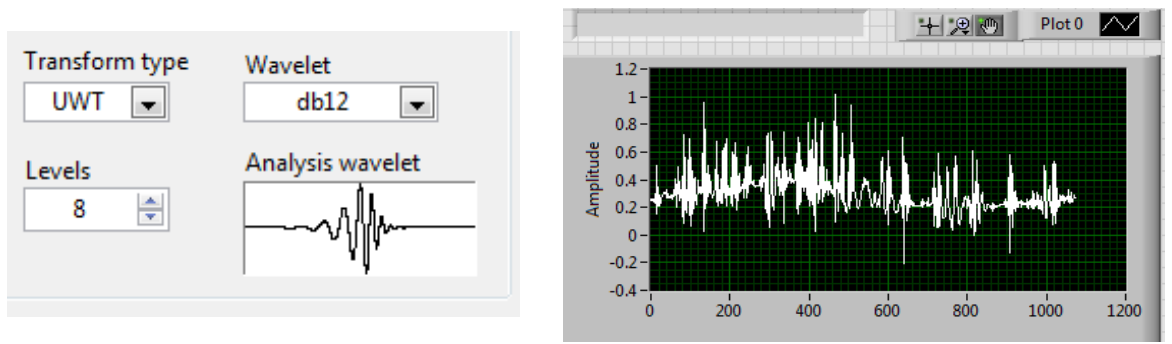

Fig. 13. Señal con el db12. 
Diseño, desarrollo y pruebas de un electrocardiógrafo virtual utilizando LabVIEW

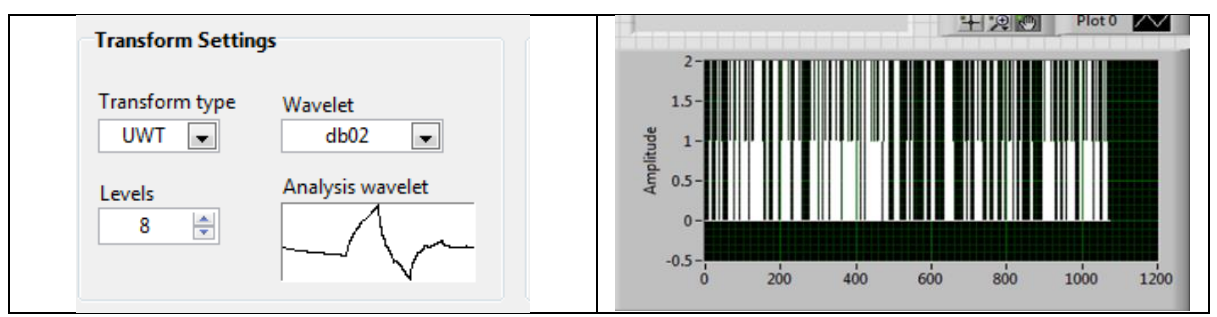

Fig. 14. Señal con el db2.

Como se puede observar en las gráficas anteriores, la selección de la Wavelet, así como la configuración de parámetros como WA Detrend, resulta crítica al realizar el tratamiento de la señal; por lo cual, realizar experimentos para la selección adecuada de estos parámetros, incrementa de manera sustancial la posibilidad de un diagnóstico en tiempo con equipos de monitorización, que aun cuando no cuentan con una estructura robusta en los requerimientos como en equipos de diagnóstico, pueden ofrecer buena calidad en sus resultados.

\section{Discusión y conclusiones}

Se observó una notable mejora con la implementación de los filtros pasa alta y pasa baja, ello permitió reducir el ruido y un análisis más exacto de la señal. Al configurar el módulo WA Detrend con los parámetros descritos en la sección 2, se eliminó la tendencia a partir de señales $1 \mathrm{D}$, ajustando los coeficientes de aproximación a ceros.

Utilizando el Waveleret Denoise se redujo el ruido en la señal, en tanto que, con el módulo Express Waveform Peak Detection se mostraron los picos característicos de una señal ECG, lo que permitió realizar un análisis detallado de la señal. En la implementación de Express Amplitude and Level Measurements, se obtuvo una señal con picos positivos y negativos, además de la distancia de pico a pico; todo ello permite ofrecer al profesional médico mayor precisión de las lecturas obtenidas para brindar diagnósticos certeros.

\section{Referencias}

1. Programa de Acción: Enfermedades Cardiovasculares e Hipertención Arterial: pp. 15-20, http://www.salud.gob.mx/unidades/cdi/documentos/enf_cardiovasculares.pdf

2. Centeno, F.: Interpretación ECG Pediátrico. Unidad de Cardiología Pediátrica, Hospital Universitario Río Hortega, http://www.aepap.org/apapcyl/ECG.pdf

3. Wells L.K: LabView Instructors's Guide. Englewood Cliffs (1995)

4. Portillo, M.: Electrocardiografía: Técnica de interpretación básica. Cardiología Infantil, Hospital Del S.E.S de Mérida (2009)

5. Park, M.: Electrocardiografia y Cardiología Pediatrica. Barcelona (2008)

6. Dubin, D.: Electrocardiografía Práctica. Madrid, Editorial Mc Graw-Hill Interamericana; $3^{\circ}$ Edición (1986)

7. Ward Ed., Walsh P. Capítulo 12, Electrocardiografía e introducción a las técnicas electrofisiológicas. $1^{a}$ Edición, Madrid, Editorial Mosby (1993) 
Guadalupe Monrroy, Dayra Zepeda, Saida Ricario, Roberto Ledesma y María Cosio

8. Ararón, L., Fernández, A.: Fisiología del Ejercicio Respuestas, entrenamiento y medición, San José, Costa Rica (1995)

9. Park, MK: Cardiología Pediátrica. Serie de manuales prácticos, Madrid (1999)

10. Vidakovic, B., Mueller, P.: Wavelets for Kids a Tutorial Introduction. Duke University, http://gtwavelet.bme.gatech.edu/wp/kidsA.pdf (1991)

11. Saavedra, G., Fernández, H., Castaño, M.: Ondeletas en ingeniería: Principios y aplicaciones. Ingeniería, investigación y tecnología, pp. 185-190, www.scielo.org.mx/scielo.php? script=sci_arttext\&pid=S1405-77432006000300005\&lng=es\&tlng=es

12. Liu, Y.H.: An ECG synchronization circuit for real-time infrared imaging of epicardial coronary vessels. Dept. of Pharmacol, Georgetown Univ. Sch. of Med., Washington, DC, USA (1993)

13. Channappa, B., Kalpana, V., Hamde, S.T., Waghmare L.M.: Estimation of ECG features using LabVIEW. International Journal of Computing Science and Communication Technologies, Vol. 2, No. 1 (2009) 